\title{
2 Motivation and Aims
}

Craniomandibular Dysfunction (CMD) has a multifactorial etiology, such as physical trauma, parafunctional habits, anxiety and stress. All these have been suggested as important initiating and perpetuating factors.

Playing a musical instrument that loads the masticatory and cervical system like the violin, has been suggested to be part of the etiological factors of CMD. Therefore an up-to-date review of the literature to assess CMD among this highly risk group will indicate the extent of this problem, give an overview of diagnostic and therapeutic methods, sensitize practitioner dentists to a more accurate understanding of the unique work conditions of violinists and of all etiologic factors caused by playing this instrument, thus making dentists able to offer preventive advice and supportive treatment to violinists, especially for those in early stages of their career.

Moreover, the analysis of the included papers aims to reveal what exactly was studied in violinists regarding to CMD, and how far manual medicine was included there. 